$71-72$

\title{
Evaluation of the sodium-glutamate blocker riluzole in a preclinical model of cervical spinal cord injury
}

Authors Yongchao $\mathrm{Wu}^{1}$, Kajana Satkundrarajah ${ }^{1}$, Yang Teng ${ }^{2}$, Diana Shu-Lian Chow ${ }^{2}$, Michael G Fehlings ${ }^{1}$ Institutions $\quad{ }^{1}$ Toronto Western Research Institute, University Health Network, University of Toronto, Canada

${ }^{2}$ College of Pharmacy, University of Houston, Texas, USA

\section{ABSTRACT}

Study type: Basic research

Introduction: Because sodium and glutamate play integrated roles in the pathology of spinal cord injury (SCI), there is intense interest in the potential role of the sodium-glutamate blocker riluzole as a neuroprotective agent for spinal cord injury. A phase I safety clinical trial of riluzole is about to commence in the USA and Canada.

Objective: The key challenges in translating riluzole to the clinic relate to uncertainty regarding the time window for post-injury administration of riluzole and the optimal dose. Hence, the current study was undertaken to study these issues in a preclinical model of cervical SCI in rodents.

Methods: Adult female rats received moderate cervical spinal cord clip compression injury $(35 \mathrm{~g} \times 1 \mathrm{~min}$ at $\mathrm{C} 7-\mathrm{Tl})$ and were randomly assigned to one of the following three groups ( $\mathrm{n}=12 /$ group $)$ : a) $8 \mathrm{mg} /$ $\mathrm{kg}$ of riluzole intraperitoneally at 1 hour (P1 group) ; b) 3 hours (P3 group) after injury or c) a control group (vehicle). The P1 and P3 groups then received $6 \mathrm{mg} / \mathrm{kg}$ of riluzole intraperitoneally every 12 hours for 7 days. A smaller group of rats $(n=10)$ received a higher dose $(8 \mathrm{mg} / \mathrm{kg}$ IP Q12 h for 7 days) of riluzole following the initial dosing. Functional recovery was tested by open field locomotion (BBB score, BBB subscore) and grid walk. Sensory function (tactile allodynia) was assessed by quantitative von Frey filament testing. Somatosensory evoked potentials (SSEP) were performed to quantitatively assess central axonal conduction. Western blotting for neurofilament 200 (NF200) was used to assess axonal integrity. Quantitative image analysis of lesion histology (HE/LFB stained sections)

Research for this paper was funded by Ontario Neurotrauma Foundation and Krembil Neuroscience Program 
was undertaken to assess tissue sparing at the injury site. High performance liquid chromatography (HPLC) was used to test pharmacokinetics of riluzole. ANOVA followed by the Tukey's post-hoc testing was used to compare the results.

Results: A total of $92.3 \%$ of injured rats that received repeated dose of $6 \mathrm{mg} / \mathrm{kg}$ riluzole survived during the term of 6 weeks, showing tolerance to this dosage. In contrast, higher doses of riluzole $(8 \mathrm{mg} / \mathrm{kg}$ IP $\mathrm{ql} 2 \mathrm{~h}$ post-injury) were associated with significant respiratory depression and high mortality (7/10 rats). Riluzole treatment (Pl group) decreased the functional deficit significantly $(P<0.05)$ at 6 weeks after injury by showing 11.4 in BBB, 3.8 in BBB subscore and 2.8 in grid walk test, compared with 10.25 in $\mathrm{BBB}, 1.1$ in BBB subscore, and 8.0 in grid walk test of control group (Figure 1). Quantitative analysis of SSEPS confirmed the neuroprotective effects of riluzole (P1 group) by showing significantly larger amplitude (control $=20.67 \mu \mathrm{V}, \mathrm{Pl}=42.67 \mu \mathrm{V}$ and $\mathrm{P} 3=37.00 \mu \mathrm{V}$, Figure 2) and reduced response latency (control $=4.05$ $\mathrm{ms}$ and $\mathrm{Pl}=3.9 \mathrm{~ms}$ ). Western blotting of NF200 indicated greater axonal preservation with riluzole treatment (Pl group) at 1 week after injury. The P3 group showed trends to improved neurobehavioral recovery and axonal preservation that did not attain significance. HPLC showed riluzole penetrated into the spinal cord as fast as 15 minutes, accumulated in the spinal cord at a concentration six times higher than in plasma, and two times higher than in brain. The drug stayed in spinal cord with a high concentration before the next injection.

Conclusions: The sodium-glutamate blocker riluzole confers neurobehavioral and anatomical neuroprotection when administered one hour after moderately severe cervical contusive/compressive injury.
Figure 1 Riluzole treatment improved functional recovery. The most robust effects were seen when the drug was injected at one hour after SCI

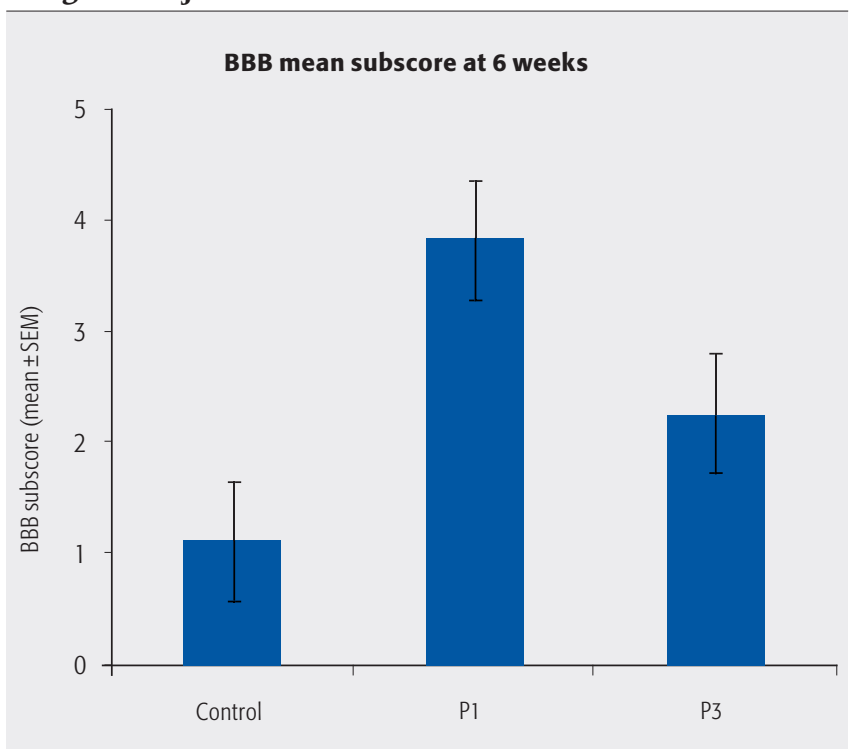

Figure 2 Riluzole treatment increased amplitude of somatosensory evoked potentials

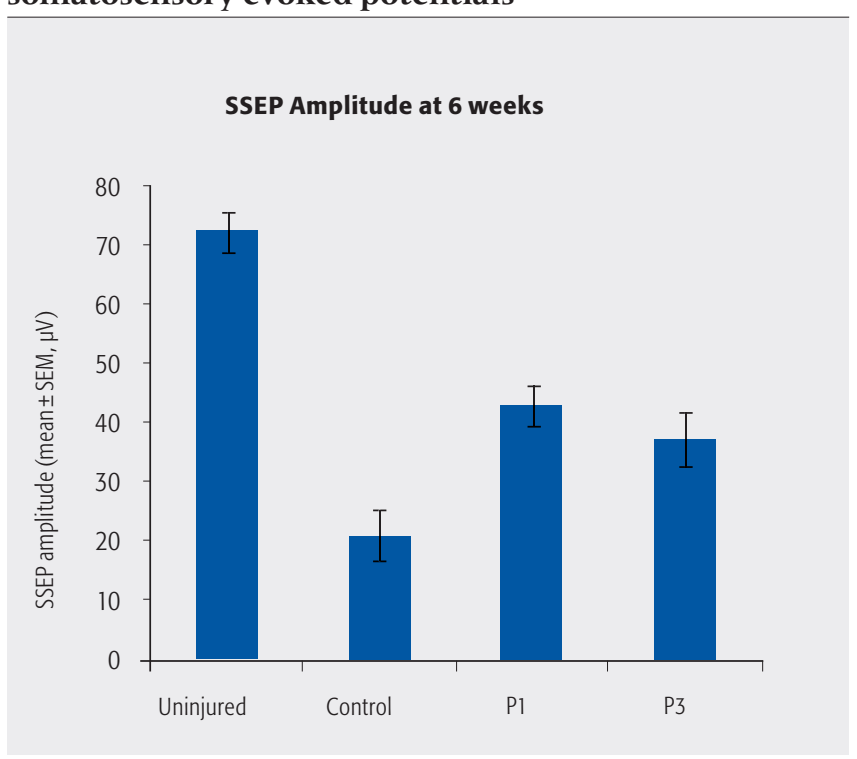

